become available, compounding practices for drug formulations in neonates should be evaluated to guarantee the correct dosing, product stability and safety.

\section{IS-031 ACUTE LIVER TOXICITY - IS IT REALLY THE PARACETAMOL? OR HOW A NEW BIOMARKER MAY AID}

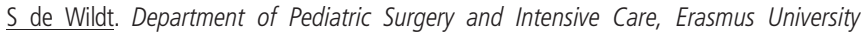
Medical Center - Sophia Children's Hospital, Rotterdam, Netherlands

\subsection{6/archdischild-2014-307384.31}

Paracetamol is the most frequently prescribed analgesic in children. Intentional and unintentional overdoses are associated with acute liver failure. Around half of all cases of acute liver failure are due to paracetamol. This led the US Food and Drug Administration this year to reduce adult dosage units of paracetamol prescription products to $325 \mathrm{mg}$ per unit and to post a Boxed Warning highlighting the potential for acute liver failure. In cases of evident paracetamol overdoses with toxic levels, the causal relationship with acute liver failure is evident, as is the case in viral hepatitis. However, in many cases of acute liver failure no underlying cause can be identified. Controversy exists as to the possibility of therapeutic doses of paracetamol to be associated with acute liver failure. Due to the complicated metabolism of paracetamol, toxicity may occur despite therapeutic doses and concentrations. Underlying disease state or co-medication may alter paracetamol metabolism resulting in increased levels of the toxic metabolite NAPQI. NAPQI binds with proteins to form protein adducts, which exert their toxic effect in liver and kidney. Recently, an assay was developed to accurately measure these adducts and subsequent research showed clear relationships with adduct levels and acute liver failure. We will illustrate the use of this new biomarker in critically ill children with acute liver failure after therapeutic doses of paracetamol.

\section{Cerebrosinovenous Thrombosis in the Newborn}

\section{IS-032 CEREBROSINOVENOUS THROMBOSIS IN THE NEWBORN: TO TREAT OR NOT TO TREAT WITH ANTICOAGULATION THERAPY}

M Moharir. Neurology, The Hospital for Sick Children, Toronto, Canada

10.1136/archdischild-2014-307384.32

Cerebral sinovenous thrombosis in the newborn: to treat or not to treat with anti-coagulation therapy Neonatal cerebral sinovenous thrombosis (CSVT) is increasingly recognised by neonatologists, paediatricians, paediatric intensivists and paediatric neurologists in recent times due to increasing awareness and improved neuroimaging techniques. Following a confirmed diagnosis of CSVT, one of the major dilemmas for the treating physician is whether or not to extrapolate Anticoagulation therapy (ACT) related data in adults and older children to newborns and initiate ACT. A major impediment for the treating physician in starting ACT is the presence of intracranial haemorrhage $(\mathrm{ICH})$ in many babies at diagnosis. Based on our current understanding of CSVT, this ICH is a result of the underlying pathophysiology of the thrombus occluding the venous system. However, due to 1) the presence of $\mathrm{ICH}$ at diagnosis and the fear of its worsening due to ACT and 2) the uncertainty of the effect of ACT on long term outcome from CSVT, most physicians are hesitant to offer ACT to the baby. But data now show that non-treatment (with ACT) of neonatal CSVT results in worsening (propagation) of the venous thrombus in nearly a third of babies and this is associated with increased risk of brain injury as well. The lecture will cover the controversies and challenges to anticoagulation therapy in neonatal CSVT and endeavour to provide a practical bedside approach to the management of CSVT in newborns.

\section{IS-033 NEURO-IMAGING IN CEREBROSINOVEOUS THROMBOSIS}

$\mathrm{L}$ de Vries, KJ Kersbergen, MJNL Benders, F Groenendaal. Neonatology, Wilhelmina Children's Hospital University Medical Center Utrecht, Utrecht, Netherlands

\subsection{6/archdischild-2014-307384.33}

Cerebral sinovenous thrombosis (CSVT) in neonates is a rare diagnosis that is increasingly being recognised with better neuroimaging techniques. Since presentation is non-specific, neuroimaging techniques are needed to confirm the diagnosis. Cranial ultrasound may suggest the diagnosis and Colour Doppler US (CDUS) can also be used for evaluation of cerebral venous sinuses in neonates. CDUS was routinely used in a large cohort of 249 asymptomatic preterm infants with a gestational age of less than 29 weeks, showing CSVT in almost 5\%, most often involvement of the transverse sinus (Raet et al Radiology, 2014). The CSVT was confirmed with MRI in the majority.

The presence of associated brain lesions, such as haemorrhagic infarction, carries a worse outcome. The spectrum of associated brain lesions recognised in neonates with CSVT, depends on their gestational age at the time of developing CSVT. Extensive white matter lesions throughout the periventricular white matter are the predominant pattern of injury associated with CSVT in symptomatic preterm infants, while an IVH associated with a predominantly unilateral thalamic haemorrhage and punctate white matter lesions (PWML) are most often seen in the full-term infant.

Routine CDUS in both preterm and full-term infants will provide more insight in the incidence of CSVT. In newborn infants admitted with seizures and/or neonatal encephalopathy CSVT should be part of the differential diagnosis. MRI including magnetic resonance venography (MRV) remains superior to cranial ultrasound and is still required in making the diagnosis of CSVT and to decide whether anticoagulation therapy should be considered.

\section{Ex-Preterm Young Adult Psychological and Social Outcomes? 1980s/90s Cohorts}

\section{IS-034 THE HEALTH, WEALTH AND SOCIAL RELATIONSHIPS OF EX VERY PRETERM/VERY LOW BIRTH WEIGHT (VP/VLBW) CHILDREN AT 26 YEARS OF AGE? THE BAVARIAN LONGITUDINAL STUDY (BEST, 1985-86 COHORT)}

D Wolke. Department of Psychology, University of Warwick, Coventry, UK

10.1136/archdischild-2014-307384.34 